\title{
The Optimization Research for the Control of Temperature on the Single- chip System Based on Particle Swarm Optimization
}

Jin Li*

School of Internet of Things, Wuxi Professional College of Science and Technology, Wuxi, Jiangsu, 214028, China

\begin{abstract}
According to the temperature measurement in the medical equipment, the activity measurement of the biological tissue should be achieved in the same environment with living human system. Aiming at the application for the temperature, a temperature controller based on the single-chip system is proposed to realize the measurement system. The modern control theory is used to analyze the controlling system which is based on the mathematic model and the state equation. The method that controls the temperature to be close to the target value and changing the parameter to reduce the error of the temperature is proposed which is based on the Particle Swarm Optimization (PSO). And the simulation results show that this method is adaptive with temperature and the controlling way is feasible. Then a single-chip system which is based on the chip PIC16C72A, is realized to solve the controlling problem. The experiments show that the scheme is equipped with accuracy and stability.
\end{abstract}

Keywords: Optimization, particle swarm optimization, single-chip, state equation, temperature measurement.

\section{INTRODUCTION}

From the year of 1980, the development of the microelectron is the most important revolution in the field of computer. The most important sign of the computer revolution is the birth of the computer embedded applications. Modern computer numerical requirements also come along with the development of the computer technology. For a long period of time, the massive computer numerical duty has developed. But the computer shows the logic operation, processing, control, attracting experts in the field of electronic controlling, the development of which requires to meet the control object requirements for embedded applications. If the massive dataprocessing computer system known as general-purpose computer system is achieved, then the system can embed objects such as ships, aircraft, motorcycles, etc., in a computer system called the embedded computer. Clearly, both the directions of technology development are different. The former requires massive data storage, handling, processing and analysis of high-speed data transmission; while the latter requires reliable operation in the target environment, the external physical parameters of high-speed acquisition, analysis and processing logic and the rapid control of external objects. It will add an early general-purpose computer data acquisition unit, where for the output driver circuit reluctance, a heat treatment furnace temperature control system is developed. A micro-computer's basic system on a chip is proposed, which is regarded as the formation of the early SCM (Single Chip Microcomputer). With the advent of a single chip in the computer industry, the industry began to result in two branches, which are: the general-purpose

*Address correspondence to this author at the School of Internet of Things, Wuxi Professional College of Science and Technology, Wuxi, Jiangsu, 214028,China; Tel: 18961759180;E-mail: 794142283@qq.com

1874-4443/15 computer systems and embedded systems. Since then, both the embedded system, and general-purpose computer systems have been developed rapidly [1].

Although the early general-purpose computer converted was into the embedded computer systems, and real embedded computer system emerged with SCM. Since the microcontroller is designed specifically for computer embedded applications, the MCU can only achieve computer embedded applications. MCU embedded applications best meet the environmental requirements such as, chip-level physical space, large-scale integrated circuits of low-cost, good peripheral interface bus and outstanding control of instruction.

With the development of the single-chip, all kinds of applications related with this kind of technology are created. For example, an industry controller can use the single-chip to realize the management of the production line. Moreover, the single-chip system is more used for solving the controlling of the smart system. In this research, a kind of singlechip named PIC16C72A is used to control the temperature to realize the measurement of the human system [2].

In medical equipment, the measurement of the biological tissue should be achieved in the environment with the same temperature. In addition, the accuracy and stability of temperature measurement pose higher requirements. Since the structure of the medical machine is limited, the temperature controller should be small. Moreover, the controller is easy to be influenced by the environmental temperature. So a special temperature measuring equipment should be designed for the special application In the following research, a measurement system is designed for such application. Based on the mathematic model of the system, the state equation is proposed for the designing of the system temperature controller. An optimization algorithm is used to optimize the parameters of the controller. The system is based on the sin- 
gle-chip PIC116C72A, and the optimization of the temperature controller is achieved by the Particl Swarm Optimization.

\section{THE HARDWARE DESIGN FOR THE TEMPERA- TURE CONTROLLING SYSTEM}

\subsection{The Design of the System Hardware}

The hardware system structure mainly consists of the single-chip, temperature sensor, incubator, input and output interface and the heating and cooling equipments. The structure of the system hardware is illustrated in (Fig. 1).

The type of the single-chip is PIC16C72A. This kind of single-chip is equipped with $5 \mathrm{~A} / \mathrm{D}$ channels which can receive the signal of the temperature sensor, with 128 bytes data storage and $2 \mathrm{~K} \times 14$ bit EPROM program storage. In addition, the temperature sensor is adopted with the BTSI-1 sensor. When the temperature is $0{ }^{\circ} \mathrm{C}$, the current signal is $273 \mu \mathrm{A}$. The temperature coefficient is $(1 \pm 0.01) \mu \mathrm{A} /{ }^{\circ} \mathrm{C}$.

The incubator is used to hold the temperature which is set by the users and the actuator is the cooling and heating equipment which tries to change the temperature in the incubator.

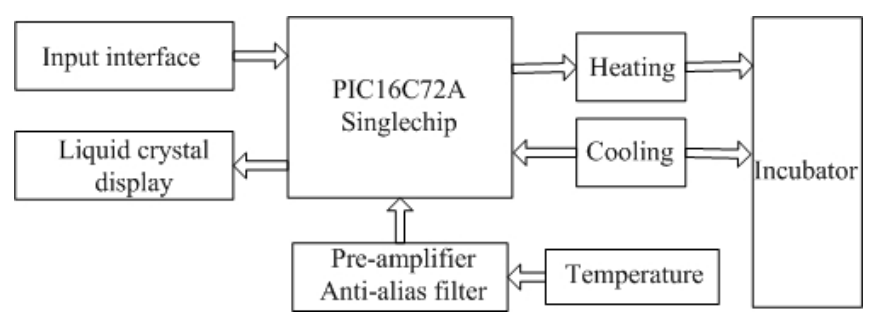

Fig. (1). The structure of the system hardware.

\subsection{The Introduction of the Work Process}

(Fig. 1) shows the work process of the temperature controlling system. The introduction of the work method is as follows. At first, the temperature sensor measures the temperature of the constant temperature box, which is the incubator. By the process of the pre-amplifier and the anti-alias filter, the output signal is treated as the feedback signal of the feedback loop. By the signal sampling of the single chip microcomputer, after digital signal processing, on one hand, the temperature of the constant temperature box can be shown on the liquid crystal display; on the other hand, the control signal is generated through the temperature control algorithm and drives the unit of cooling or heating the air in the constant temperature box. The constant temperature box keeps the temperature stable according to the target temperature of the user input from the keyboard. The controlled object is the system which consists of the unit of the cooling/heating and constant temperature box system. The temperature sensor, preamplifier and mixed filter form a feedback loop.

\section{THE MATHEMATICAL MODEL AND SYSTEM CONTROL SCHEME}

\subsection{The Mathematical Model of the System}

The control system can be divided into four parts. They are: cooling/heating unit, incubator, temperature sensor, pre-amplifier and anti-alias filter. The transfer functions are $G_{1}, G_{2}, G_{3}, G_{4}$. Based on the experiences, these transfer functions can be treated as the first order damp elements. The transfer functions are as follows.

$$
\begin{cases}G_{1}=\frac{T_{u}(s)}{u(s)}=\frac{k_{p 1}}{t_{1} s+1} & G_{2}=\frac{T(s)}{T_{u}(s)}=\frac{k_{p 2}}{t_{2} s+1} \\ G_{3}=\frac{u_{1}(s)}{T(s)}=\frac{k_{p 3}}{t_{3} s+1} & G_{4}=\frac{u_{2}(s)}{T_{u}(s)}=\frac{k_{p 4}}{t_{4} s+1}\end{cases}
$$

In the expression, $u(t)$ is the output control signal of the single-chip. $T_{u}(t)$ is the temperature wind of the incubator. $T(t)$ is the temperature of the incubator. And $u_{1}(t)$ is the temperature signal of the temperature sensor. $u_{2}(t)$ is the output after the signal is processed by the pre-amplifier and the anti-alias filter. $T_{u}(s), T(s), u_{1}(s)$ and $u_{2}(s)$ are the Lars transforms. The parameter $s$ is the complex frequency in the Lars transform. Moreover, the gains of the first order damp elements and the time constants are as follows according to the calculation and experiments.

$$
\left\{\begin{array} { l l } 
{ k _ { p 1 } = 0 . 5 } & { k _ { p 2 } = 1 } \\
{ k _ { p 3 } = 0 . 5 } & { k _ { p 4 } = 0 . 5 }
\end{array} \left\{\begin{array}{cl}
t_{1}=20 \mathrm{~s} & t_{2}=160 \mathrm{~s} \\
t_{3}=12.5 \mathrm{~s} & t_{4}=0.2 \mathrm{~s}
\end{array}\right.\right.
$$

In this way, the state function of the system is calculated in the following expression.

$$
\dot{x}=\left[\begin{array}{c}
\dot{T}_{u}(t) \\
\dot{T}(t) \\
\dot{u}_{1}(t) \\
\dot{u}_{2}(t)
\end{array}\right]=\left[\begin{array}{cccc}
-\frac{1}{20} & 0 & 0 & 0 \\
\frac{1}{160} & -\frac{1}{160} & 0 & 0 \\
0 & \frac{1}{12.5} & -\frac{1}{12.5} & 0 \\
0 & 0 & 5 & -5
\end{array}\right] \cdot\left[\begin{array}{c}
T_{u}(t) \\
T(t) \\
u_{1}(t) \\
u_{2}(t)
\end{array}\right]+\left[\begin{array}{c}
0.05 \\
0 \\
0 \\
0
\end{array}\right] \cdot u(t)
$$

$=A x+B u$

In this expression, the parameters $\dot{T}_{u}(t), \dot{T}(t), \dot{T}(t)$, $\dot{u}_{2}(t)$ are the derivatives of state variables $T_{u}(t), T(t)$, $u_{1}(t), u_{2}(t) . A$ is the coefficient matrix and $B$ is the input matrix.

$x=\left[\begin{array}{llll}T_{u}(t) & T(t) & u_{1}(t) & u_{2}(t)\end{array}\right]^{\mathrm{T}}$ is the state vector. And $\dot{x}$ is the derivative of $x$. Considering the equations above, the initial condition of the control system is described in Eq. (4).

$\left\{\begin{array}{cl}T_{u}\left(t_{0}\right)=t_{\min } & T\left(t_{0}\right)=t_{\text {min }} \\ u_{1}\left(t_{0}\right)=t_{\text {min }} & u_{2}\left(t_{0}\right)=t_{\text {min }} \\ t_{\min } \leq T_{u}(t) \leq t_{\max }\end{array}\right.$

In these conditions, $t_{0}$ is the initial beginning time. $t_{\text {min }}$ and $t_{\max }$ are the lowest and highest temperature of the wind, 
respectively, which are outputted by the cooling or heating unit. The values of them are normally decided by the environment temperature and the highest heating power of the unit.

The output equation is as follows. In this equation, $y$ is the output vector. $y_{1}(t)$ is the temperature in the incubator. $y_{2}(t)$ is feedback signal of the output. $C$ is the output matrix.

$$
y=\left[\begin{array}{l}
y_{1}(t) \\
y_{2}(t)
\end{array}\right]=\left[\begin{array}{llll}
0 & 1 & 0 & 0 \\
0 & 0 & 0 & 1
\end{array}\right] \cdot\left[\begin{array}{l}
T_{u}(t) \\
T(t) \\
u_{1}(t) \\
u_{2}(t)
\end{array}\right]=C x
$$

And the target function is shown in Eq. (6). In the equation, the parameter $t_{e}$ is the end time when the calculation of the target function is over. $T_{d}$ is the target temperature which is given by users.

$J=\frac{1}{2} \int_{t_{0}}^{t_{e}}\left(T(t)-T_{d}\right)^{2} d t$

According to the principles of modern control theory, the control system is fully equipped with the features of the controllability and observability based on the state equation and the output equation of the system.

\subsection{The Design of the System Control Algorithm}

Based on the feature of the temperature controlling system, a usual control method is that several temperature stages are set in the range of the target temperature. The relationship between the control signal $u$ and the measuring value $u_{2}$ is defined as Eq. (7).

$$
u=\left\{\begin{array}{c}
T_{\max }, \quad u_{2} \leq u_{2} *-\frac{\Delta}{2} \\
T_{\max }-\frac{u_{2}+\Delta-u_{2}{ }^{*}}{\Delta}\left(T_{\max }-T_{\min }\right),-\frac{\Delta}{2} \leq u_{2} \leq u_{2}{ }^{*}+\frac{\Delta}{2} \\
T_{\max }, u_{2} \geq u_{2}{ }^{*}+\frac{\Delta}{2}
\end{array}\right.
$$

In the above equation, $u_{2}{ }^{*}$ represents the measuring value according to the target temperature $T_{d}$, and $\Delta$ is the range of the scale.

But since the control signal can only take the maximum or minimum value of the range, in order to make the heating unit output between the highest and lowest temperature of hot air, control signals must be given by the method of solid state relay frequency control. For the control of PWM wave, each cycle is controlled by pulse width a heating pulse number. This method cannot realize the output of a specific tem- perature of hot air directly, but can guarantee the relative distance of hot air temperature $[3,4]$.

According to the measuring temperature $u_{2}, T$ the can be estimated. The discretization of state space equation can be defined as follows.

$$
T_{k}=\frac{\left(1+\frac{t_{3}+t_{4}}{T_{s}}+\frac{t_{3} t_{4}}{T_{s}^{2}}\right) u_{2, k}-\left(\frac{2 t_{3} t_{4}}{T_{s}^{2}}+\frac{t_{3}+t_{4}}{T_{s}}\right) u_{2, k-1}+\left(\frac{t_{3} t_{4}}{T_{s}^{2}}\right) u_{2, k-2}}{K_{3} K_{4}}
$$

In Eq. (8), $T_{d}$ is the sample period. $u_{2, k}$ is the $k$ th value of the sampling. The control signal is decided by the error between the $T_{d}$ and $T_{k}$. In this way, the system control accuracy can be improved.

When the value of $\Delta$ is very big, temperature can be stable very rapidly, but the highest temperature and the lowest temperature of heating and cooling temperature have a great influence on the last temperature in the control process. The final temperature is not accurate. When the $\Delta$ value is small, the final temperature is accurate. Nevertheless the control process has a long transition. Moreover, when the value $\Delta$ is very small, there will be a significant oscillation. So we can use the mathematical model of system simulation, to calculate the value of the objective function based on Eq. (6), and choose the best $\Delta$ value that can make the target function $J$ minimum.

Because of the feature that when $\Delta$ is very big, the control process is stable, a corrected parameter $\delta$ is introduced in the control algorithm. Eq. (9) is the changed control scheme and the accuracy of the control system can be achieved by adjusting the value of $\delta$. Parameter $\Delta$ is the optimization variable in the following optimization algorithm The objective function is $J$.

$$
u=\left\{\begin{array}{c}
T_{\text {max }}, T_{k} \leq\left(T_{d}+\delta\right)-\frac{\Delta}{2} \\
T_{\text {max }}-\frac{T_{k}+\frac{\Delta}{2}-\left(T_{d}+\delta\right)}{\Delta}\left(T_{\text {max }}-T_{\text {min }}\right),\left(T_{d}+\delta\right)-\frac{\Delta}{2} \leq T_{k} \leq\left(T_{d}+\delta\right)-\frac{\Delta}{2} \\
T_{\text {max }}, T_{k} \geq\left(T_{d}+\delta\right)+\frac{\Delta}{2}
\end{array}\right.
$$

\section{OPTIMIZATION AND SIMULATION RESULTS}

\subsection{The Introduction of the Particle Swarm Optimiza- tion}

The optimized mathematical model, the restrain and objective function have been decided. Based on the amounts of GA experiments and PSO simulation, three or more improvements are needed to solve the single-objective-function with multivariate, multiple peak value and multi-constraint.

1) Avoid prematurity: For GA, because of the variance of individual distribution, population size etc., there will be local convergence problems. The PSO used in this 
study is based on the roulette wheel selection operator and makes the population memory less at initial phase to avoid local optimal solution. Thus, the prematurity problem is avoided excellently

2) Rate of convergence: PSO has perfect memory for global optimal solution. Each generation of particles through self-learning and social-learning utilizes the inheritance of themselves, at the same time, particles move to global optimal solution more quickly. So the rate of convergence of PSO is faster than GA's.

3) Optimal solution available: Upon the acquisition of solution, GA gets into prematurity easily, and the solution obtained is quasi-optimal, not the best. The PSO can make global optimal solution available.

For the three aspects, PSO is selected to optimize the parameter of driver and search the optimal solution.

\subsection{The Standard Particle Swarm Optimization}

The iterative formula of standard PSO is as follows:

$V_{i}(\mathrm{t}+1)=X_{i}(\mathrm{t})+\omega V_{i}(\mathrm{t})+\mathrm{C}_{1} r_{1}\left(\mathrm{P}_{\mathrm{i}}(\mathrm{t})-X_{i}(\mathrm{t})\right)+$

$\mathrm{C}_{2} \mathrm{r}_{2}\left(\mathrm{P}_{\mathrm{g}}(\mathrm{t})-X_{i}(\mathrm{t})\right)$

$\mathrm{P}_{\mathrm{i}}(\mathrm{t})$ is the individual extreme, $\mathrm{P}_{\mathrm{g}}(\mathrm{t})$ is the global extreme. Both $C_{1}$ and $C_{2}$ are constants which represent the degree of influence by social cognitive knowledge and the particle individual cognition (also known as the learning rate), respectively. $C_{1}$ and $C_{2}$ are usually set to the same value which weigh equally. $r_{1}$ and $r_{2}$ are the random numbers on the interval of $(0,1)$. As an inertial factor, $\omega$ can dynamically adjust the iterative velocity of the particles over time so that the particles can gradually move to the local search [5]. And the standard process of particle swarm algorithm is shown in (Fig. 2).

And $\Delta$ is the target optimization variable. The range of the parameter can be easily decided in the range of $[0,100]$ by the particle swarm optimization. According to the experience, the size of the particle swarm is usually set to 20-40.In this research, the size of the group is set to 30 and the terminated generations $\mathrm{T}$ are set to 50 . Generally, the maximum velocity is set to $10 \%-20 \%$ of the interval. In this study, $V_{\max }$ is $10 \%$. If the current velocity is bigger than the maximum, the current velocity is updated to the maximum. Inertia weight factor $\omega$ is 0.7 which describes the flight direction of particles. Considering that the accelerated factor is often set to $2-4$, both $C_{1}$ and $C_{2}$ are set to 2.5 which describe the weight of self-learning based on the individual memory and the weight of self-learning and relationship between the particles respectively [6].

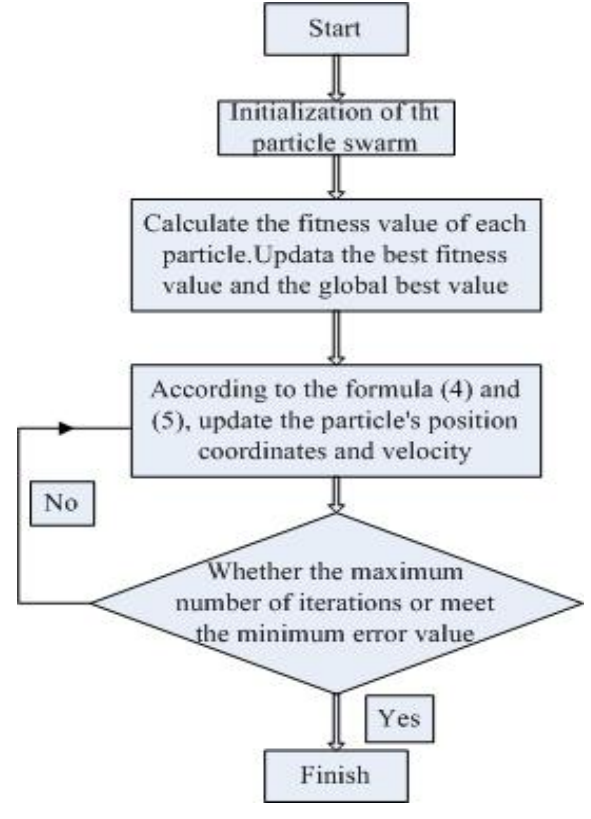

Fig. (2). The standard process of PSO.

For the fitness calculation of each particle, it is considered that the values of objective function must be greater than zero. Therefore, the fitness function is set as shown in following Eq. (11) based on the Eq. (6).

According to the problem, it belongs to the problem of searching the minimum value.

fit $\{J\}=\frac{1}{100+J}$

The objective function value of excellent particle is small. Correspondingly; its fitness function value is large which can lead the particle motion to the optimal solution [7, 8].

\subsection{The Simulation Results of PSO}

According to the selected optimization method and the operating parameters, the scale range parameter $\Delta$ is matched based on the particle swarm optimization. The optimization results are as follows;

The distribution of the fitness function values of the particle for the initial particle group is shown in (Fig. 3).

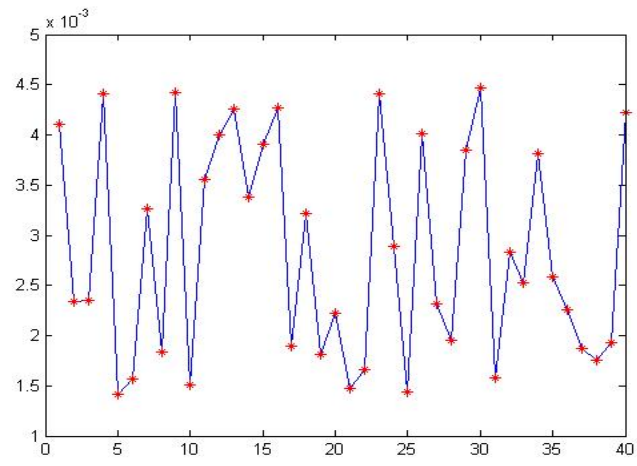

Fig. (3). Distribution of fitness function values for the initial particle group. 
And the distribution of fitness function values of particles after 50 iterations is shown in (Fig. 4).

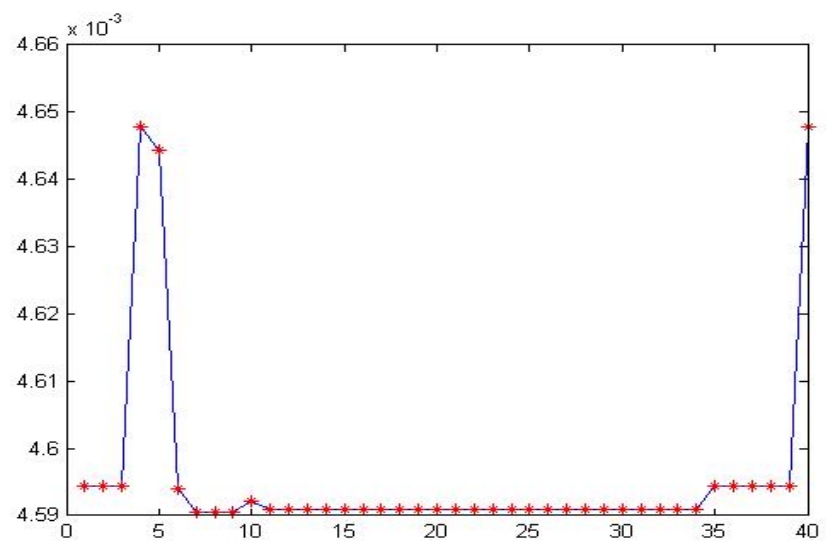

Fig. (4). Distribution after 50 iterations.

The distribution of the fitness function value for the optimal solution in the process of 50 iterations is illustrated in (Fig. 5).

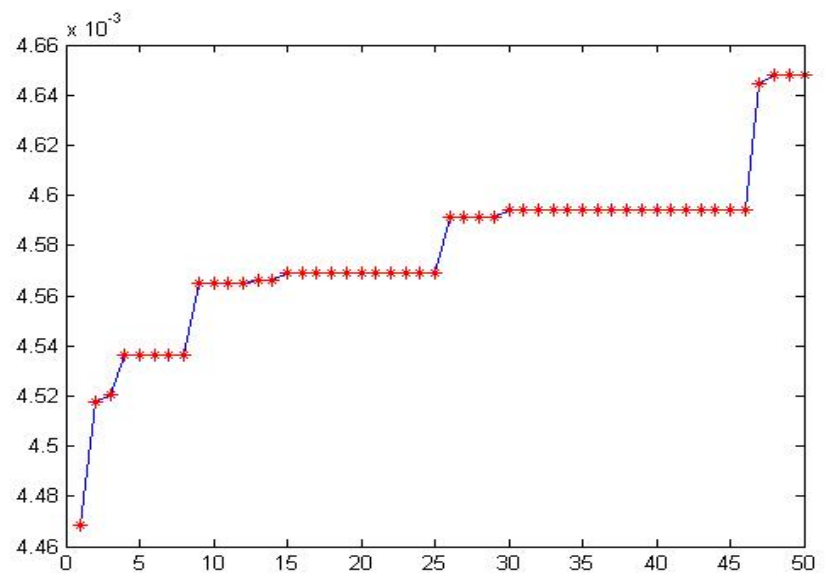

Fig. (5). Distribution for the optimal solution.

The experiments show that the distribution of the fitness function value for the initial particles is dispersive and highly random that shows the diversity of particle swarm which is good for searching the optimal solution (Fig. 3). As shown in (Figs. 4 and 5), the fitness function value of particle swarm tends to unite after 20 iterations. But there are still a small portion of the particles in local solution space. The fitness function value converges better and tends to be more stable basically after 50 iterations. Above all, particle swarm optimization algorithm has a good effect on solving the problem of parameter matching.

And at last, the optimization of parameter $\Delta$ is calculated to be 3.5 ,therefore, the simulation of the temperature controlling based on the single-chip is achieved. The simulation experiment is designed under the assumption that the target temperature is 35 and the environment temperature is 25 in the first $20 \mathrm{~min}$ and later the temperature becomes 10 . The simulation result is shown in (Fig. 6).

The optimization results have good control effects which make the system more stable and accuracy.

\subsection{The Actual Control Results based on the Single-chip}

The following experiment results are based on the real control by the single-chip PIC16C72A, and the value of the initial temperature is 25 . The control target is 35 . The transient in the first 15 minutes of the thermostatic control is illustrated in (Fig. 7).

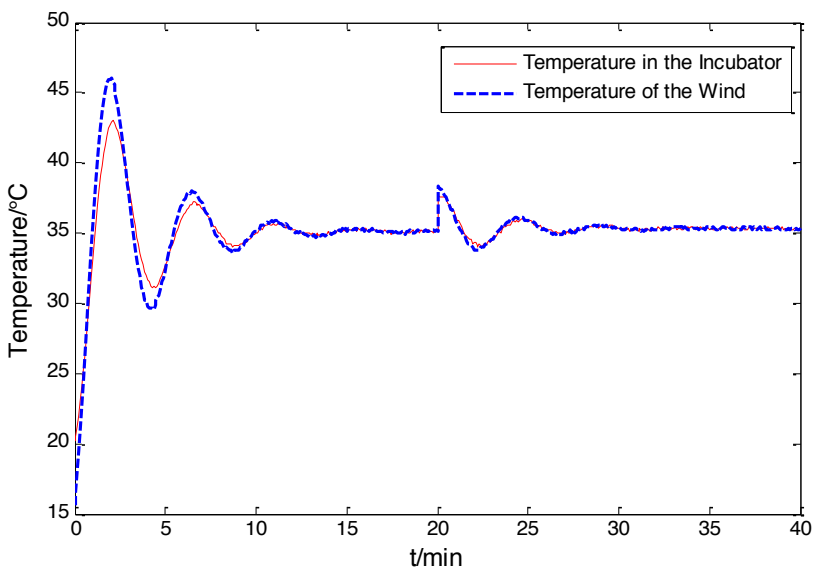

Fig. (6). The simulation results of the thermostatical control.

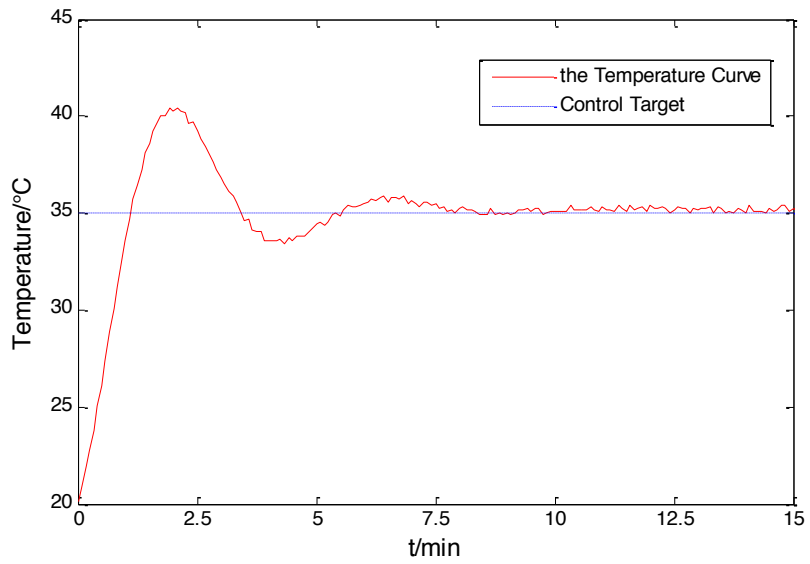

Fig. (7). Transient in the first 15 minutes of the thermostatical control.

\section{CONCLUSION}

Based on the mathematical model and the state space equation which reflect the actual situation of system, the simulation calculation can be achieved onthe basis of selecting control scheme and parameters. The control scheme of temperature control system proposed in the research has good control effects which are optimized by the particle swarm optimization, and the influence of environment temperature is smaller than the normal system. The temperature controller in heat preservation condition is not very good, and easily affected by ambient temperature under the condition of constant temperature control.

\section{CONFLICT OF INTEREST}

The author confirms that this article content has no conflict of interest. 


\section{ACKNOWLEDGEMENTS}

Declared none.

\section{REFERENCES}

[1] U. C. Moon, and K.Y. Lee, "Temperature control of glass melting furnace with fuzzy logic and conventional PI control", In: Proceedings of the American Control Conference, 2000, pp. 2720-2724.

[2] P. J. Gawthrop, and P. E. Nomikos, "Adaptive temperature control of industrial process a comparative study", IEEE Proceedings, vol. 137, pp. 137-144, 2008.

[3] M. Mezmaz, N. Melab, Y. Kessaci, Y.C. Lee, E.G. Talbi, A.Y. Zomaya, and D. Tuyttens, "A parallel bi-objective hybrid metaheuristic for energy-aware scheduling for cloud computing systems", Journal of Parallel and Distributed Computing, vol. 71, pp. 14971508,2011
[4] W. Li, "The research of forward solution for six degrees of freedom parallel mechanism," Huazhong University of Science and Technology Library, vol. 25, no. 4, pp. 38-40, 1997.

[5] J. Long, "Optimization design of brushless dc motor speed control system based on genetic algorithm," Journal of Civil Aviation Flight University of China, vol. 16, no. 5, pp. 21-24.

[6] W. Luo, Research and Implementation of the Three Degrees of Freedom Motion Platform, M.s thesis, Huazhong University of Science and Technology, China, 2011.

[7] M. Saravanan, and S. M. Raja, "Application of particle swarm optimization technique for optimal location of FACTS devices considering cost of installation and system load ability," Electric Power Systems Research, vol. 77, pp. 276-283, 2007.

[8] H.I. Shaheen, G.I. Rashed, and S.J. Cheng, "Application and comparison of computational intelligence techniques for optimal location and parameter setting of UPFC," Engineering Applications of Artificial Intelligence, vol. 23, pp. 203-216, 2010.

Received: June 16, 2015

(C) Jin Li.; Licensee Bentham Open.

This is an open access article licensed under the terms of the (https://creativecommons.org/licenses/by/4.0/legalcode), which permits unrestricted, non-commercial use, distribution and reproduction in any medium, provided the work is properly cited. 